\title{
A flash based thermal simulation of scanning paths in LPBF additive manufacturing
}

\author{
Kamel ETTAIEB $^{1}$, Sylvain LAVERNHE ${ }^{1, a}$, and Christophe TOURNIER $^{1}$ \\ ${ }^{1}$ Université Paris-Saclay, ENS Paris-Saclay, Institut Farman (FR3311), LURPA, 91190 Gif-sur-Yvette, France \\ ${ }^{a}$ Corresponding author, Email adress: sylvain.lavernhe@ens-paris-saclay.fr
}

\begin{abstract}
During manufacturing by Laser Powder Bed Fusion (LPBF), the laser-scanning path influences the thermomechanical behavior of parts. Therefore, it is necessary to validate the path generation considering the thermal behavior induced by this process to improve the quality of parts. The purpose of this study is to develop an analytical thermal 3D model that allows the efficient analysis of laser trajectories in each layer. First, the proposed approach consists of applying impulse temperature excitation according to the trajectory, then summing the thermal conduction effects in the material to calculate the distribution of the temperature field in the part at each time step. The developed model is calibrated and validated by comparison with thermal results obtained by FEM software as well as experimental measurements. Numerical investigation is performed to compare different scanning path strategies on the Ti6Al4V material with different simulation parameters. The simulation results confirm the effectiveness of the approach as well as the influence of trajectories on the maximum temperature field and the distribution of thermal gradients during the manufacturing of each layer.
\end{abstract}

Keywords: additive manufacturing, LPBF, scanning path, thermal modeling, thermal gradient.

\section{Introduction}

The process of Laser Powder Bed Fusion (LPBF) is a technique of direct manufacturing that uses a high-power laser to melt powder according to the scanning paths. During this process, the temperatures in local areas could generate significant thermal gradients. These gradients lead to the presence of residual stresses that affect the mechanical characteristics of the part and may cause deformation, as well as micro- and macro-cracks due to the cooling conditions [1].

In this context, scanning paths play a major role in the fusion and solidification of the material, and therefore, of the part. In the literature, several scanning approaches have been proposed; among them, the zigzag filling approach is the most widely used. It is simple to implement in a trajectory calculation algorithm but presents many drawbacks, such as heat accumulation at the extremities where the movement inversions are located [2]. This leads to the appearance of residual stresses, and consequently, to the deformation of the part or even the generation of cracks [3]. To solve these problems, the sequenced scanning path has been proposed, during which the laser first scans the surface in alternate lines and then returns to fill the remaining spaces. Experimental results show that sequenced scanning produces parts with small deformations and almost no shrink- age, compared with parts obtained by continuous scanning [4]. Indeed, in the case of continuous scanning, there is not enough time to dissipate the heat.

The generation of filling scanning paths given the contour-offset method is an approach that has demonstrated efficiency through the improvement in the geometric accuracy and homogenization of the stress distribution [5]. Through a different method, Chantarapanich et al. [6] proposed a honeycomb strategy. The main drawback of this approach is the large number of hexagonal shapes, and thus, geometric discontinuities that limit the scanning speed and increase the manufacturing time. Jhabvala et al. [2] developed the sectoral trajectory whose principle is to divide the surface into small sectors. Each area is scanned with the strategy of parallel lines in alternating directions. This method modifies the thermal dynamics and limits the inhomogeneity of the temperature field, which reduces the level of residual stresses on the part [7]. Ma and Bin tested the fractal trajectory [8] in which the scanning path is generated according to the Hilbert curve. The simulation of this trajectory shows a uniform distribution of thermal gradients, and consequently, a decrease in the residual stresses compared with the zigzag trajectory.

All these studies have demonstrated the major role of the scanning strategy, which affects the geometric quality and mechanical properties of the parts, as well as the efficiency of the process in 
terms of speed and productivity. Therefore, it is essential to be able to simulate the thermal behavior to validate the influence of these trajectories on the temperature distribution during manufacturing and on the appearance of part defects.

In general, to study the residual stresses in parts manufactured by LPBF, the temperature distribution during manufacturing must first be simulated. For that purpose, several models at the macroscopic and microscopic levels have been developed [9]. Temperature evolution is often obtained by finite-element methods. Criales et al. [10] proposed a two-dimensional (2D) model to analyze the influence of material properties and process parameters on the temperature field for Inconel 625. Hussein et al. [11] used 3D finite element simulations to study temperature fields and residual stresses in 316L stainless steel layers, built in LPBF without support. The studies of Li et al. [12], Nickel et al. [13] and Foroozmehr and Kovacevic [14] present 3D models based on the finite element method as well. These works focused mainly on the evolution of temperature, residual stresses, and warping of parts manufactured by LPBF, according to the scanning strategies. They showed that the type of trajectory, lengths of scanning paths, and their orientations can play a crucial role in the level of residual stresses in the part. Indeed, the best strategy is the one that slows down the cooling cycle and reduces the temperature gradient.

Other analytical and semi-analytical models were proposed in the literature. These models are generally based on the Rosenthal's solution originally designed for welding field [15]. Promoppatum et al. [16] used this approach to predict the evolution of the temperature field and the dimensions of the melt pool during the manufacturing of a part of Inconel 718. The thermal results obtained from the analytical resolution show that the dimensions of the melt pool and the cooling rate are more sensitive to the material absorptivity. They also mentioned the effects of simplifying assumptions related to the Rosenthal solution whose advantage lies in its simplicity. Plotkowski et al. [17] used a 3D model developed in the field of laser welding which enables the prediction of the macroscopic variation of the temperature in the part. However, this solution is based on the hypothesis that the heat source has a circular ring shape, which is not representative of energy distribution in the case of the LPBF process. The principle of the model proposed by Forslund et al. [18] consists of discretizing the trajectory according to small segments and integrating each segment separately using the Gaussian quadrature method whose order depends heavily on the process parameters. The downside of their approach is the need to iteratively test the quadrature orders under various conditions and to pre-calculate a lookup table for later use. In addition, the segmentation into segments makes it suitable for the high scanning speeds of the EBM process, but less relevant for the LPBF process. Yang et al. [19] proposed a semi-analytical model to calculate the temperature evolution along a given scanning path. The proposed approach consists of superposing analytical solutions for point heat sources and complementary numerical fields to impose the boundary conditions. This approach gives satisfactory results. However, these two techniques are usually used separately, and their coupling can further increase the computation time. In addition, the study has not validated the impact of boundary conditions using finite differences on the accuracy of the thermal calculation.

Regardless of the simulation technique used (numerical or analytical), the simulation of the LPBF process requires relatively small temporal and spatial steps to predict the thermal gradients that are generated during melting. However, maintaining such small discretization parameters increases the computation time and the memory size reserved for the simulation. Therefore, the purpose of this study is to develop an analytical thermal model that enables the fast analysis of the scanning paths for each manufacturing layer while respecting as much as possible the characteristics of the LPBF process. The proposed approach allows the implementation of adjustment solutions that lead to reduce the computation time and the memory occupancy.

The rest of the paper is organized as follows: the model and its numerical implementation are presented in the second section. The third section is dedicated to the validation of the model in the case of Ti6Al4V alloy by aligning the model with the 3D finite element method, conducted with the reference software proposed by ESI [20]. The proposed methods to optimize the computation time are presented in the fourth section. The fifth section is dedicated to the numerical investigation of the influence of scanning paths on the temperature distribution and thermal gradients. Finally, the paper is concluded in Section 6.

\section{Thermal modeling}

\subsection{Physical description of the model}

The difficulty in controlling the LPBF process comes from the complexity of the different phenomena involved in the melting of the powder and its solidification. Indeed, as shown in Fig. 1, several physical behaviors occur at different scales: fluid mechanics (e.g., surface tension, gravity, and buoyancy), metallurgy (phase change), and heat transfer.

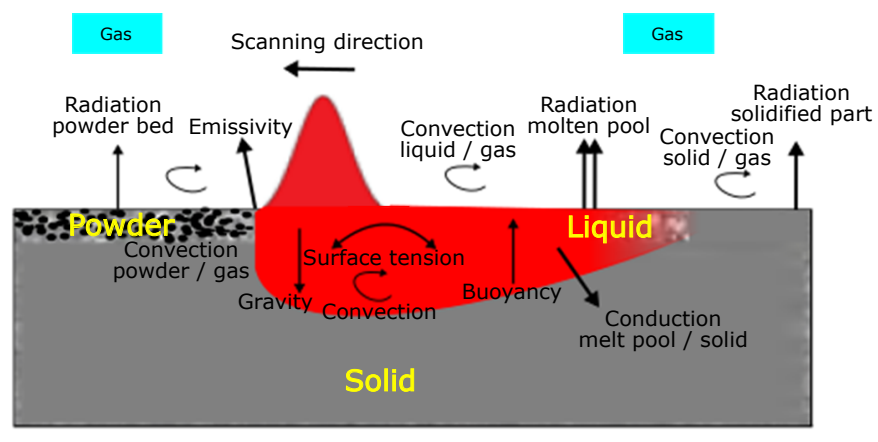

Figure 1: Physical phenomena involved in the LPBF process

During the LPBF process, when the laser beam irradiates the surface of the powder bed, its energy is transferred in several ways: a fraction of the energy is absorbed by the powder, while the rest is reflected. The absorbed energy increases the temperature of the 
material beyond its melting point. Another part of the remaining energy is transferred by conduction in the part or lost either by convection or radiation from the surface to the environment.

The spatial and temporal distribution of the temperature field is defined by the heat equation Eq. 1 .

$$
\rho C \frac{\partial T}{\partial t}=\lambda \nabla^{2} T+Q
$$

where $\rho$ is the material density $\left(\mathrm{kg} \mathrm{m}^{-3}\right), C$ is the specific heat capacity $\left(\mathrm{J} \mathrm{kg}^{-1} \mathrm{~K}^{-1}\right), T$ is the temperature $(\mathrm{K}), \lambda$ is the thermal conductivity $\left(\mathrm{W} \mathrm{m}^{-1} \mathrm{~K}^{-1}\right)$, and $Q$ is the heat source generated by the laser $\left(\mathrm{W} \mathrm{m}^{-3}\right)$.

Considering $n$ as the normal vector to the interface, the boundary condition at the top surface of the powder is written as follows (Eq. (2)):

$$
-\lambda \frac{\partial T}{\partial n}=q-q_{c}-q_{r}
$$

where $q$ is the laser surface flux $\left(\mathrm{W} \mathrm{m}{ }^{-2}\right), q_{c}$ is the heat lost by convection $\left(\mathrm{W} \mathrm{m}^{-2}\right)$ and $q_{r}$ is the heat losses by radiation $\left(\mathrm{W} \mathrm{m}^{-2}\right)$.

The powder bed is heated to a preheating temperature equal to $T_{0}$ at $t=0$.

In this study, some assumptions are adopted:

1. The conductivity $\lambda$ and the absorptivity $A$ are those of the consolidated material which is a common hypothesis in LPBF [18]. Indeed, on the one hand, powder is a porous medium that absorbs more energy than solid (around $70 \%$ versus $30 \%$ ) and on the other hand the thermal conductivity of the solid is higher than powder (around 21 versus $7 \mathrm{~W} \mathrm{~m}^{-1} \mathrm{~K}^{-1}$ at $1900 \mathrm{~K}$ ) [21], [22]. Therefore, shortly after the laser-matter interaction, the evolution of the thermal behavior in both media, over time, could be considered as similar.

2. Physical properties of materials are independent of the temperature.

3. Radiation $q_{r}$ and convection $q_{c}$ losses are considered to be null.

4. Phase change and the latent heat are not taken into account in the simulation.

In this context, the thermal problem of powder melting is reduced to a conduction problem after the interaction between the laser and material. To solve this problem, an explicit formulation of the evolution of the temperature in the part over time is proposed.

\subsection{Presentation of the Flash method}

The Flash method is commonly used to measure the thermal diffusivity of materials [29]. The idea consists of loading a plate of thickness $L$ and radially semi-infinite on its front surface by impulse excitation $\delta(t)$ (Dirac function), which has a Gaussian distribution of radius $R$. Considering $z$ as the downward vertical axis, $r$ the radial direction, $z=0$ represents the top surface and $r=0$ the center of the laser spot. Then, its impulse response in temperature is measured on the irradiated surface. The sample is supposed to be homogeneous, isotropic, and opaque. The mathematical interpretation of these phenomena leads to the following differential equations [23]:

$$
\nabla^{2} T(r, z, t)=\frac{1}{\alpha} \cdot \frac{\partial T(r, z, t)}{\partial t}
$$

where $\alpha$ is the thermal diffusivity $\left(\mathrm{m}^{2} \mathrm{~s}^{-1}\right)$. For the top surface $(z=0)$ :

$$
-\lambda \frac{\partial T(r, z=0, t)}{\partial z}=\frac{2 A Q_{l}}{\pi R^{2}} \cdot e^{\frac{-2 r^{2}}{R^{2}}} \delta(t)
$$

where $R$ is the radius of the laser beam (m), $A$ is the absorptivity of the material, and $Q_{l}$ is the laser energy $(\mathrm{J})$.

On the opposite surface of the plate, a constant heat flow is defined as follows (Eq. (5)):

$$
-\lambda \frac{\partial T(r, z=L, t)}{\partial z}=0
$$

The laser energy is calculated as follows (Eq. (6)):

$$
Q_{l}=P \cdot \Delta f
$$

where $\Delta f$ is the elementary duration (s) of the laser emission.

The analytical resolution of the equation (Eq. (3)) leads to the calculation of the thermal effect of a flash $\left(T_{f}\right)$, which is written as follows (Eq. (7)):

$$
T_{f}(r, z, t)=\frac{2 A Q_{l}}{\varepsilon \sqrt{\pi^{3} t}} \cdot \frac{1}{R^{2}+8 \alpha t} \cdot e^{\frac{-2 r^{2}}{R^{2}+8 \alpha t}} \cdot \sum_{n=-\infty}^{\infty} e^{\frac{-\left[(n-1) L+\frac{z}{2}\right]^{2}}{\alpha t}}
$$

where $\varepsilon$ is the thermal effusivity ( $\mathrm{J} \mathrm{K}^{-1} \mathrm{~m}^{-2} \mathrm{~s}^{-\frac{1}{2}}$ ), which is calculated as a function of the other thermal parameters of the material (Eq. (8)):

$$
\varepsilon=\sqrt{\lambda \cdot \rho \cdot C}
$$

The parameter $t_{0}$ represents the lower bound of the validity domain of the model [23]. In general, its value must satisfy the following condition (Eq. (9)):

$$
t_{0} \geq 10 \cdot \Delta f
$$

This method allows the calculation of the temperature evolution in the part over time after an infinitely short thermal excitation on the surface, which is characterized by a Gaussian distribution. An example obtained, at different times, for a titanium alloy (Ti6Al4V), is shown in Fig. 2. The dashed curves represent thermal responses outside the range of validity. Thus, following a flash, the resulting temperature profile becomes less intense and larger over time. This behavior reflects the radial diffusion of energy. Table 1 lists the process parameters and material specifications used in the rest of the paper. 


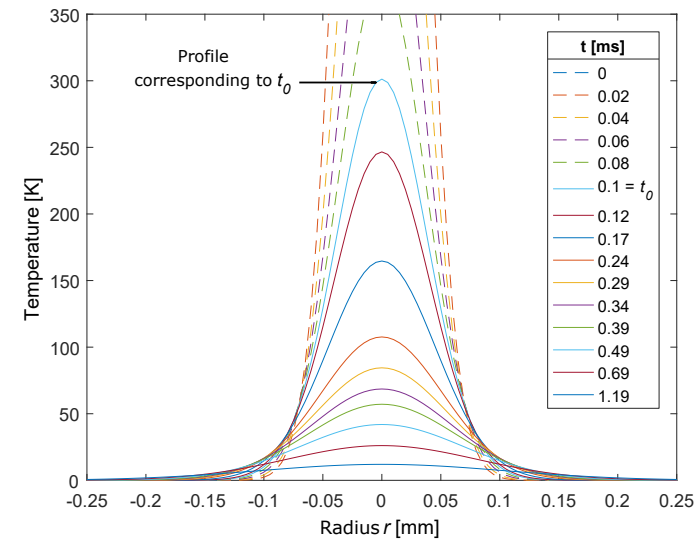

Figure 2: Simulated surface temperature profiles for a flash

Table 1: Simulation parameters

\begin{tabular}{ll|l}
\hline$P$, laser power & {$[\mathrm{W}]$} & 300 \\
$R$, effective radius of laser beam & {$[\mathrm{m}]$} & $5.10^{-5}$ \\
$V$, scanning speed & {$\left[\mathrm{m} \mathrm{s}^{-1}\right]$} & 1 \\
$A$, absorptivity & {$[\%]$} & 30 \\
$\lambda$, thermal conductivity & {$\left[\mathrm{W} \mathrm{m}^{-1} \mathrm{~K}^{-1}\right]$} & 15 \\
$C$, specific heat capacity & {$\left[\mathrm{J} \mathrm{kg}^{-1} \mathrm{~K}^{-1}\right]$} & 800 \\
$\rho$, density & {$\left[\mathrm{kg} \mathrm{m}^{-3}\right]$} & 4420 \\
$\varepsilon$, thermal effusivity & {$\left[\mathrm{J} \mathrm{K}^{-1} \mathrm{~m}^{-2} \mathrm{~s}^{-\frac{1}{2}}\right]$} & 7282 \\
$T_{0}$, preheating temperature & {$[\mathrm{K}]$} & 293 \\
$\Delta f$, temporal step & {$[\mathrm{s}]$} & $10.10^{-6}$ \\
\hline
\end{tabular}

\subsection{Simulation methodology}

The thermal effect of flash $T_{f}$ can be considered the impulse response of the part. Thus, it is defined by the convolution product with Dirac excitation $\delta(t)$ (Eq. (10)):

$$
\left(T_{f} * \delta\right)(r, z, t)=\int_{-\infty}^{+\infty} T_{f}(r, z, \tau) \cdot \delta(t-\tau) \mathrm{d} \tau
$$

Fig. 3a shows the thermal response following the application of a flash on the surface at $t=\tau$.

The method used to simulate the thermal behavior of the LPBF process consists of converting the laser scanning path with a given speed as a succession of impulse solicitations (Fig. 3b). Thus, the irradiation of the laser on the powder according to the scanning path is expressed by the Heaviside function $\mathscr{U}(t)$ (Eq. (11)):

$$
\mathscr{U}(t)= \begin{cases}0, & t<0 \\ 1, & t \geq 0\end{cases}
$$

This function reflects the state of the laser, which takes on the value of 1 during the ignition phase.

The global temperature response $T_{g}$ at instant $t$ and on each point of the part defined by coordinates $(x, y, z)$ is the cumulative sum

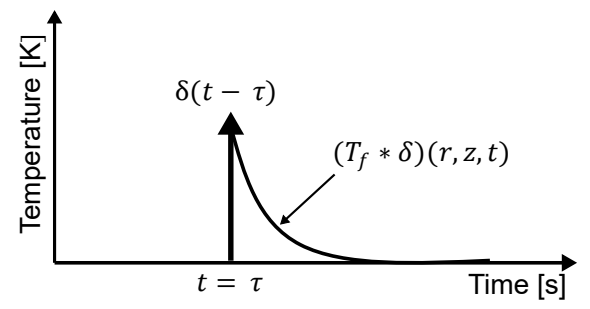

(a)

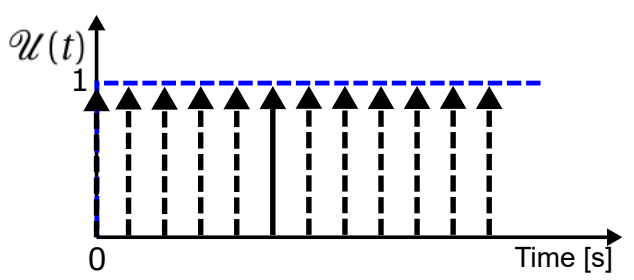

(b)

Figure 3: (a) Thermal response of an elementary flash, (b) impulse excitation modeling a scanning path

of the impulse responses given the path scan up to that moment. Mathematically, it corresponds to the convolution product between the function $\mathscr{U}(t)$ and the thermal effect $T_{f}$ of an elementary flash that moves on the surface according to the path (Fig. 4).

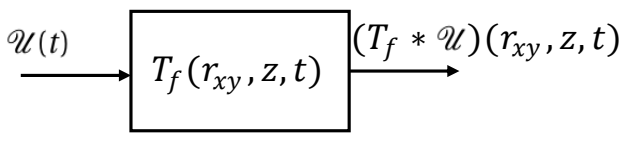

Figure 4: Global thermal response to a succession of Dirac excitations

Considering also the preheating temperature of powder $T_{0}$, the global temperature $T_{g}$ at each point $(x, y, z)$ according to time $t$ is expressed by Eq. (12):

$$
T_{g}(x, y, z, t)=T_{0}+\int_{-\infty}^{+\infty} T_{f}\left(r_{x y}, z, t-\tau\right) \mathscr{U}(\tau) \mathrm{d} \tau
$$

where $r_{x y}$ represents the distance between each flash center and the $(x, y)$ point. The discretized form of Eq. (12) with temporal step $\Delta t$ can be written as follows (Eq. (13)):

$$
T_{g}(x, y, z, t)=T_{0}+\sum_{i=0}^{n} T_{f i}\left(r_{i}, z, t-i . \Delta t\right) \quad \text { with } n=\left\lfloor\frac{t}{\Delta t}\right\rfloor
$$

The spatial discretization of the scanning path $d l$ is calculated as a function of temporal step $\Delta t$ and scanning speed $V$ (Eq. (14)):

$$
d l=V \cdot \Delta t
$$

Once the discretization of the scanning path is carried out, the principle consists of applying a flash on each point of the trajectory (Fig. 5) in order to calculate the global temperature response in 
each point of the part and at each time step. The methodology for calculating the temperature field in the part, for a given scanning strategy, can be summarized in the diagram shown in Fig. 6. Therefore, the principle consists of summing the thermal effects of the laser at each point of the scanning path to calculate the distribution of the temperature field in the part at each time step.

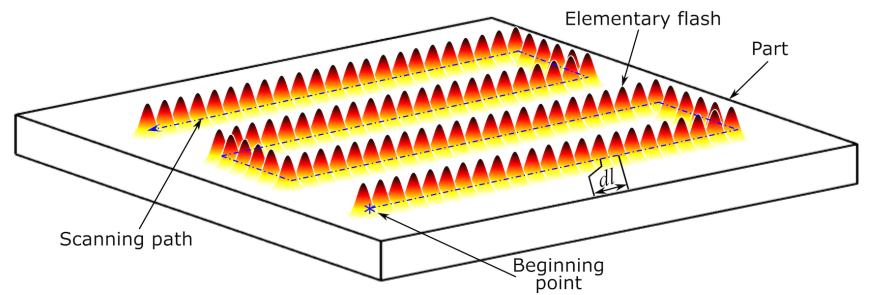

Figure 5: Principle of scanning path simulation by the Flash method

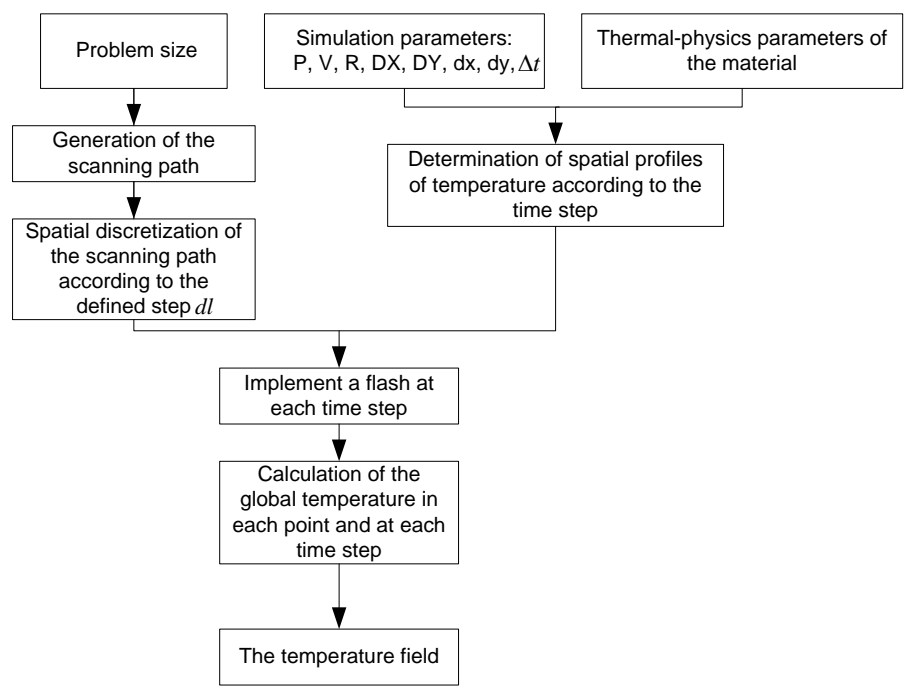

Figure 6: Methodology of Flash simulation

\section{Validation of the Flash method}

\subsection{Model calibration}

This step consists of aligning the developed model with 3D FEM simulation conducted by a renowned software program proposed by ESI (Engineering Systems International) [20]. Both simulations are performed on a segment-type path (Fig. 7a) for the alignment.

The tracking of the temperature at sensor 1 with the two models shows a difference between the two simulations. Indeed, since the flash model does not natively integrate phase changes, the temperatures reached with this model are higher and the associated cooling
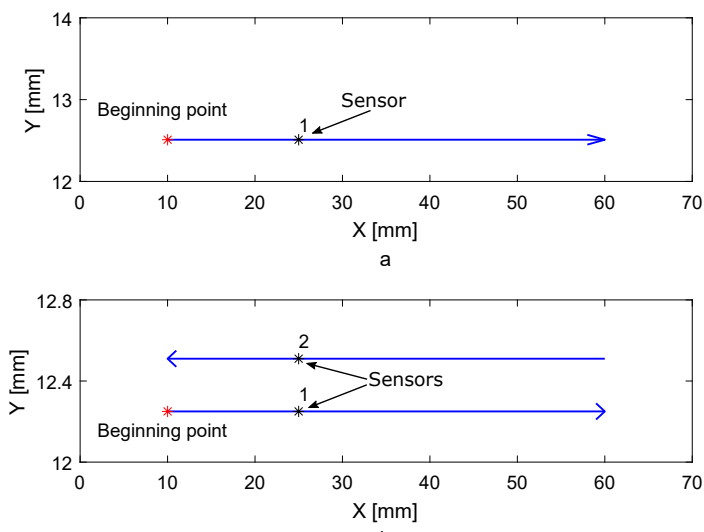

Figure 7: (a) Tested trajectories of segment type and (b) zigzag

cycles are longer. (Fig. 8). In order to calibrate the Flash model, an additional coefficient $\gamma$ is assigned to the energy transferred to the material (Eq. (15)). Thus, the assumptions of the Flash model mentioned above are taken into account and compensated. Within the case of study and associated parameters defined in Table 1 this coefficient has been set to 0.4 .

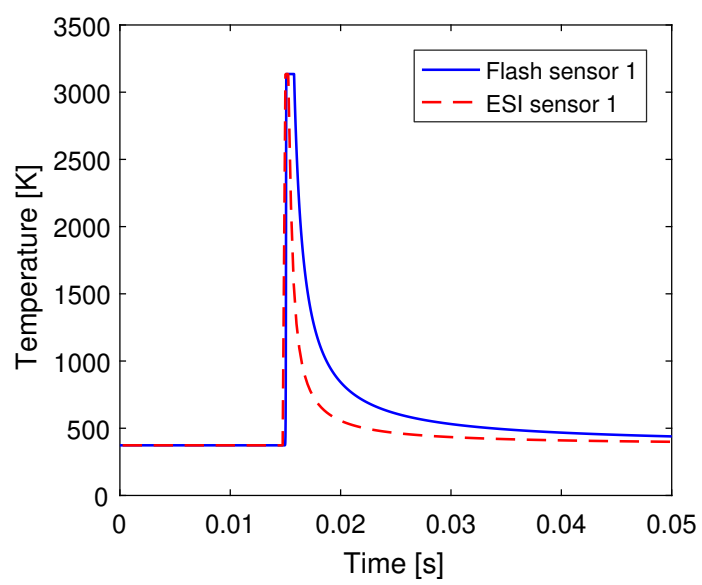

Figure 8: Temperature variation at sensor 1 before calibration

$$
T_{g}(x, y, z, t)=T_{0}+\gamma \sum_{i=0}^{n} T_{f}\left(r_{i}, z, i . \Delta t\right)
$$

The alignment of the Flash model with respect to the ESI model provides a good similarity between the temperature profiles for all points. The curves in Fig. 9 show the temperature variation at sensor 1 with the two simulations. The results show a clear convergence in behavior during thermal cycles, and heating and cooling phases.

Once the alignment has been performed, the simulation (Fig. 10) validates the model on sensors 1 and 2 for a zigzag path (Fig. 7b). The results of the Flash method and those of the ESI simulation show very similar temperature variation curves (Fig. 10). The large 


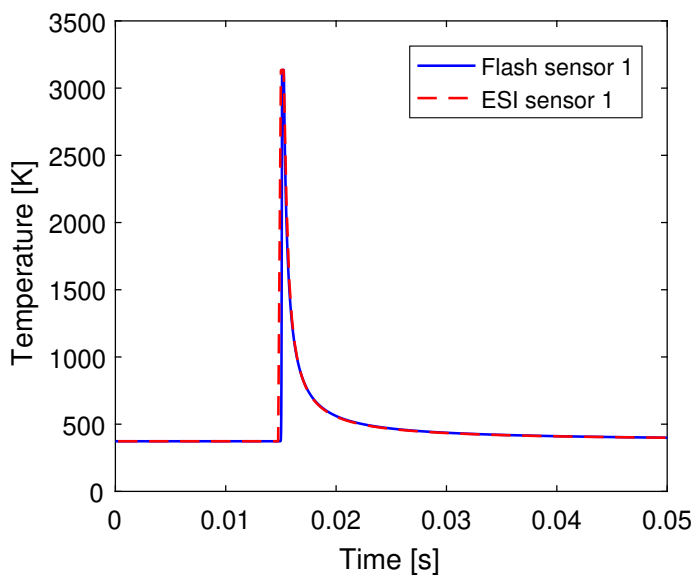

Figure 9: Temperature variation at sensor 1 after calibration

peaks are similar, especially where the laser passes over the sensors. The simulated temperatures measured at a point radially distant from the laser passage show slight differences. This fact is illustrated by the small peaks in the curves in the zooms of Fig. 10. This phenomenon reflects a shortcoming of the Flash method in capturing radial heat dissipation. Indeed, the maximum error measured is equal to $50 \mathrm{~K}$. This value has no significant effect on the simulation, as it is detected in a point far from the focus area where there is a large thermal change. The simulations also show a quick increase in temperature. This demonstrates that, during laser passage, the heat transfer according to the scanning direction is low compared to the radial dissipation. It is clear that the laser speed is higher than the speed of heat propagation in the scanning direction.

\subsection{Simulation application}

To illustrate the simulation results on a small parallelepipedic part, the case of a straight horizontal path (Fig. 11) is used with following data configuration:

1. part size: $2 \times 0.5 \times 0.15 \mathrm{~mm}^{3}$

2. length of the scanning path: $18 \mathrm{~mm}$

3. temporal step $\Delta t: 10 \mu \mathrm{s}$

4. spatial steps $d x$ and $d y$, which represent the spatial discretization step of an elementary flash: $10 \mu \mathrm{m}$

5. discretization steps $D X, D Y$ and $D Z$, which represent the spatial discretization step of the part: $10 \mu \mathrm{m}$

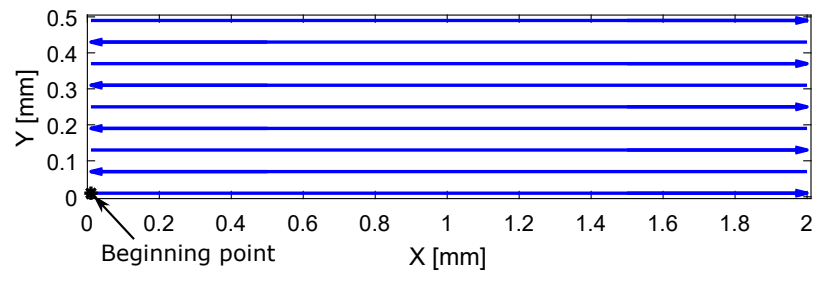

Figure 11: Zig-zag path

The Flash model is implemented in Matlab and run on a laptop computer equipped with an Intel Core i5 6200U @ $2.3 \mathrm{GHz}$ (2 cores) and $8 \mathrm{~GB}$ of RAM.

The global temperature maps on the upper surface of the part at two different instants are shown in Fig. 12a and 12b. The simulations show that the temperatures are locally higher than the melting temperature of the material. The melt pool moves with the heat source. The plots also show a heat trail, with a nearly elliptical shape, which becomes increasingly large as the laser moves and the temperature increases. The simulated temperatures inside the melt pool can reach very high values, beyond the evaporation temperature of the material $(3500 \mathrm{~K})$, because the proposed model does not include phase change or, thus, latent heat of fusion or evaporation. Therefore, temperatures are locally capped to the evaporating temperature.

Fig. 12c represents the part temperature field at $t=9 \mathrm{~ms}$ and Fig. 12d represents the same temperature field in a cross-section perpendicular to the scanning direction, at the same instant and for $X=1 \mathrm{~mm}$. The simulation shows the temperature variation according to $z$.

The curve presented in Fig. 13a describes the temperature distribution along the A-A section on the upper surface and the dashed line indicates the laser center position at the considered instant. It can be observed that the temperature level is higher where the powder has been melted and lower in the upstream area along the trajectory. Indeed, the heat dissipation in the scanning direction is low due to the scanning speed which is greater than the heat propagation speed. The melt pool length is equal to $552 \mu \mathrm{m}$. The simulation also shows that the maximum temperature value is recorded a little behind the laser beam center. This effect is consistent with the results from the literature [24].

Temperature profile along the B-B section is shown in Fig. 13b. It can be seen that the temperature distribution along the scanning line is not symmetrical with respect to the center of the laser spot. The heat in solidified areas, where the laser is previously passed, is not completely dissipated. The melt pool width is equal to $129 \mu \mathrm{m}$. This dissymmetry could be amplified in the powder side by considering the real conductivity of the powder as a function of temperature (assumptions 1 and 2).

Temperature distribution along the $\mathrm{C}-\mathrm{C}$ section is presented by the curve in Fig. 13c. Temperature profile following the depth gives the main interest of the 3D simulation. The simulation shows, as expected, that the maximum temperature is located on the upper part surface. This also shows that the thermal gradient is important 

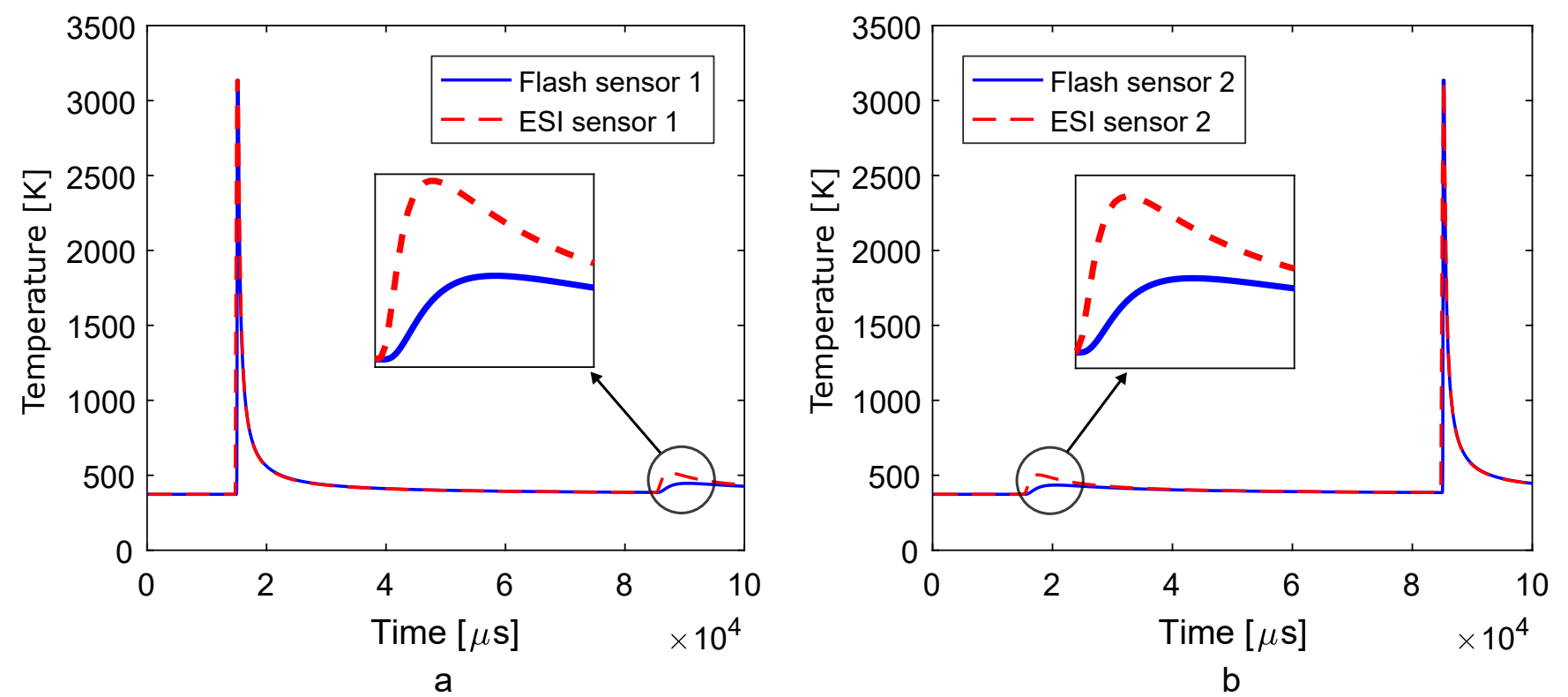

Figure 10: Temperature variation at sensors 1 and 2

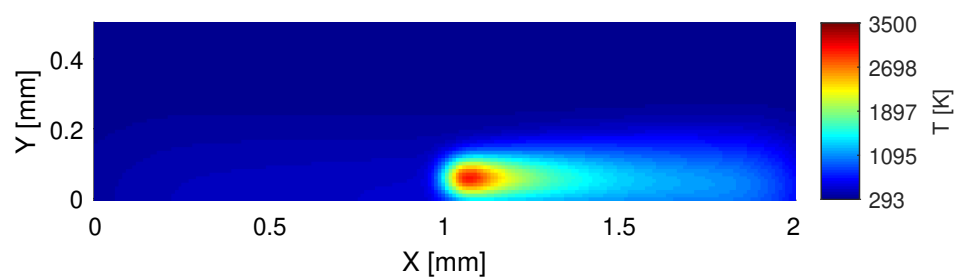

(a) Temperature map on the top surface at $t=3.06 \mathrm{~ms}$

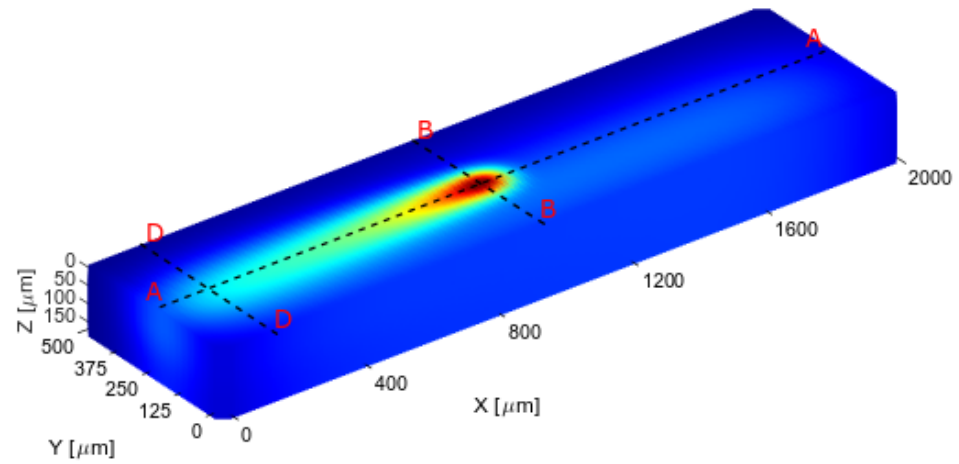

(c) Temperature map in the part at $t=9 \mathrm{~ms}$

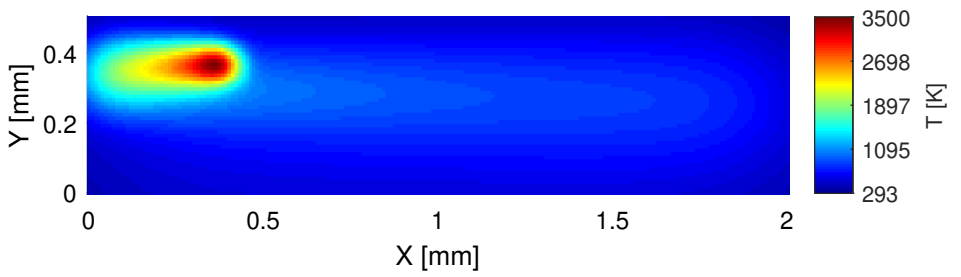

(b) Temperature map on the top surface at $t=12.49 \mathrm{~ms}$ Temperature $[\mathrm{K}]$

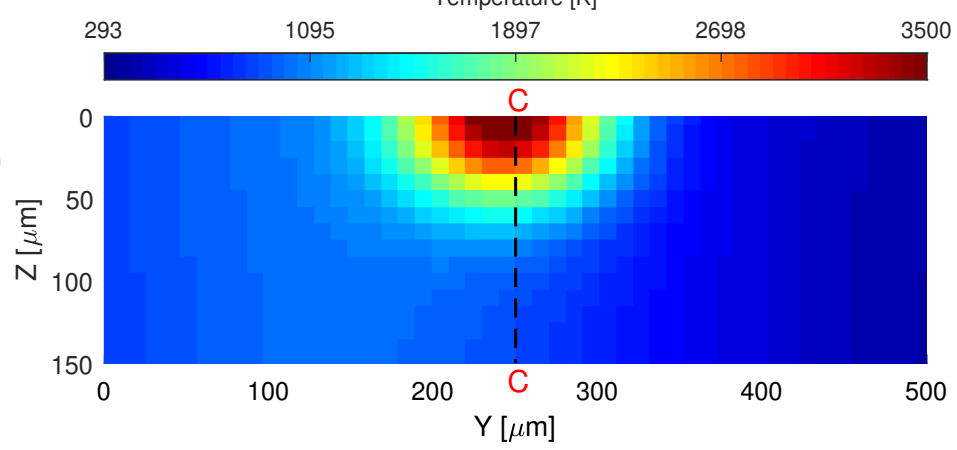

(d) Cross-section $X=1 \mathrm{~mm}$ and at $t=9 \mathrm{~ms}$

Figure 12: Temperature maps

and exceeds $2.10^{7} \mathrm{~K} \mathrm{~m}^{-1}$. The depth of the melted area is equal to $52 \mu \mathrm{m}$. As a result, this simulations enables to predict part of the previous layer is remelted. The characteristic dimensions (length, width and height) of the melt pool are calculated thanks to the melting temperature iso surface in the 3D mesh. Fig. 14 shows the evolution of the melt pool dimensions along a scanning line (A-A section). The width of the melt pool is important at the beginning of the line where it reaches $163 \mu \mathrm{m}$. Then, it decreases to reach about
$115 \mu \mathrm{m}$ as the laser advances (Fig. 14a). The melt pool also has a greater depth at the beginning of the line than at the end (Fig. 14b). The melt pool depth exceeds the thickness of a layer $(40 \mu \mathrm{m})$ and reaches a maximum value equal to $70 \mu \mathrm{m}$. Indeed, in the case of the zig-zag path, the jump from the line $n$ to the line $n+1$ does not leave enough time for the material to dissipate heat and cool down. Consequently, the melting is developed in a preheated area which increases the dimensions of the melt pool. 


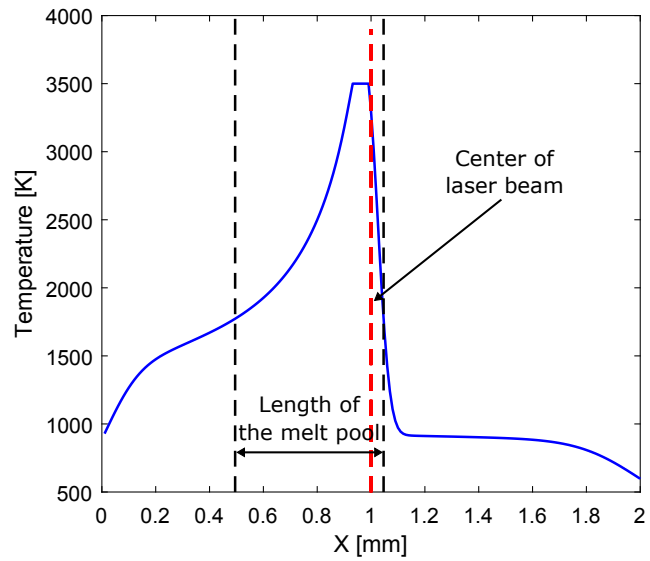

(a) $\mathrm{X}$ direction (A-A dashed line)

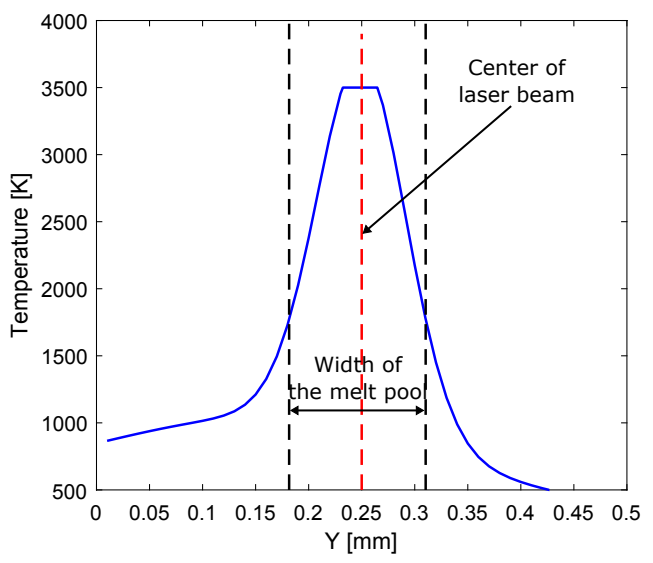

(b) $\mathrm{Y}$ direction (B-B dashed line)

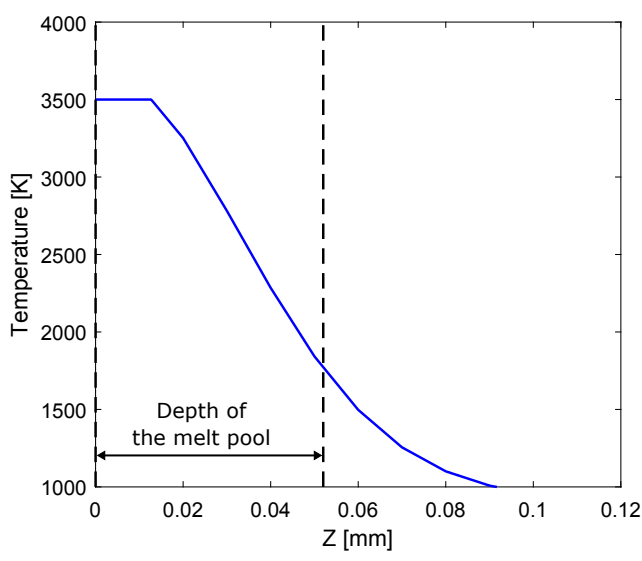

(c) $\mathrm{Z}$ direction (C-C dashed line)

Figure 13: Temperature profiles according to the three spatial directions

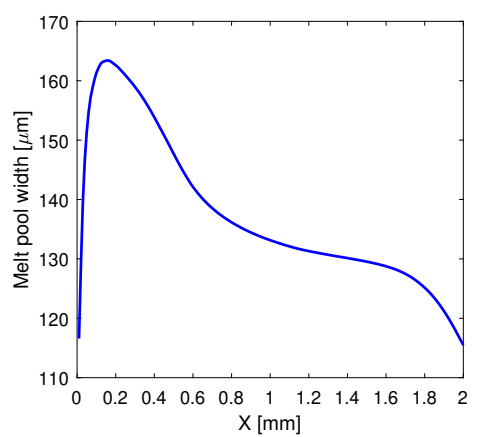

(a)

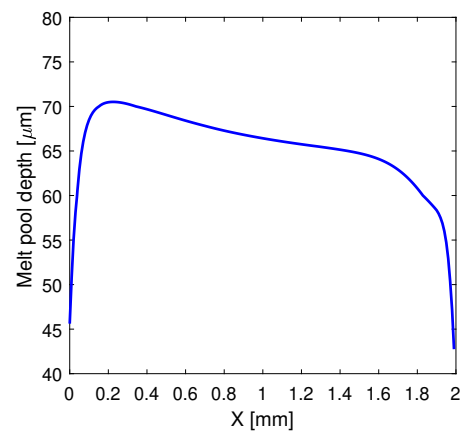

Figure 14: Evolution of the melt pool geometry ((a): width - (b): depth)

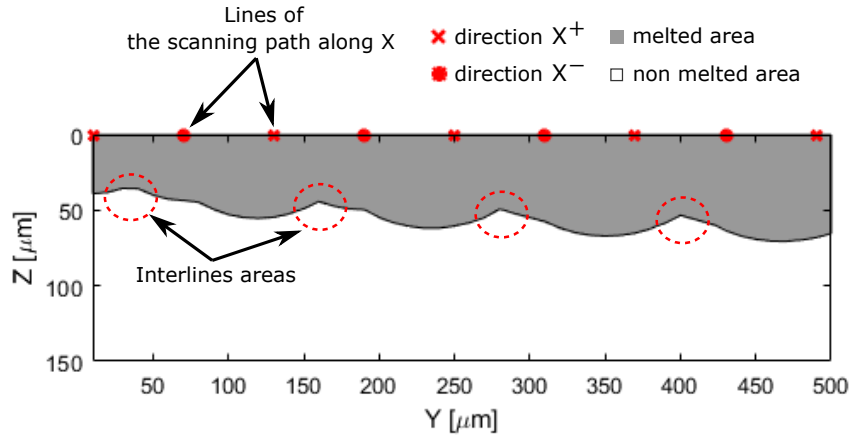

Figure 15: Cross-section of the part at $X=0.25 \mathrm{~mm}$

\subsection{Experimental validation}

The purpose of this section is to compare the results of the Flash method with experimental results from the literature and more particularly the work of Dilip et al. [25] about the variation of the width and depth of a single bead according to the process parameters.

In this study, the absorption coefficient $A$ of Ti6Al4V alloy has been set at $40 \%$. Indeed, this value has been used in the works of Oleak et al. [26], or Gharbi et al. [27]. Some of the experimental data from [25] is first used to shift the amount of energy actually transferred to the part surface in the Flash method based on the associated assumptions (section 2). The data used are the width and depth of the melt pool for a straight path with a laser power of $150 \mathrm{~W}$ and velocities of $0.5 \mathrm{~m} \mathrm{~s}^{-1}, 0.75 \mathrm{~m} \mathrm{~s}^{-1}, 1.0 \mathrm{~m} \mathrm{~s}^{-1}$ and $1.2 \mathrm{~m} \mathrm{~s}^{-1}$. The dimensions of the simulated part are $1 \mathrm{x} 0.5 \mathrm{x}$ $0.15 \mathrm{~mm}^{3}$. Fig. 16 shows the evolution of the width and depth of the melt pool for the different tested scanning speed values. The experimental results are described by the red curves whereas the simulation results before alignment are shown by the black curves. The comparison between the results shows a difference with all the scanning speed values. In order to calibrate the Flash model, the correction coefficient is set at 1.2. The simulation results, after alignment, 


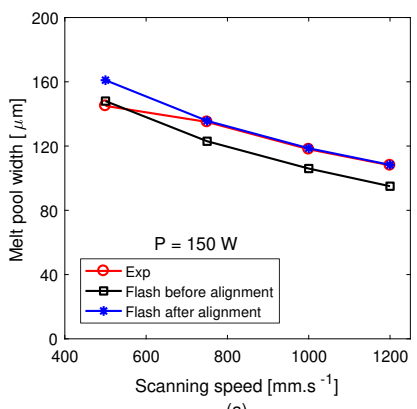

(b)

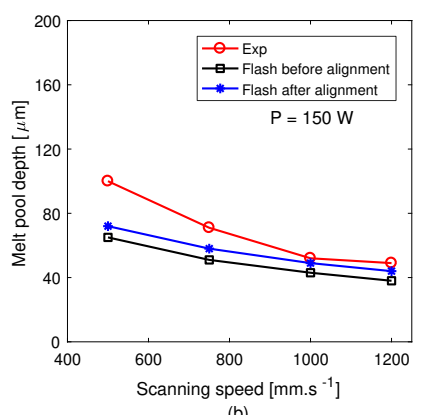

Figure 16: Flash model calibration, $P=150 \mathrm{~W}$

are presented by the blue curves. The comparison results of the width show a good similarity with scanning speeds of $0.75 \mathrm{~m} \mathrm{~s}^{-1}$, $1 \mathrm{~m} \mathrm{~s}^{-1}, 1.2 \mathrm{~m} \mathrm{~s}^{-1}$. However, a $11 \%$ difference has been registered when the scanning speed is equal to $0.5 \mathrm{~m} \mathrm{~s}^{-1}$ (Fig. 16a). The simulated depths are closer (Fig. 16b). The maximum difference is equal to $28 \%$ with a scanning speed of $0.5 \mathrm{~m} \mathrm{~s}^{-1}$ and it reaches $5 \%$ with $1 \mathrm{~m} \mathrm{~s}^{-1}$.

Once the calibration has been performed, the flash simulation is compared with the experimental results obtained by Dilip et al. [25] with a power value of $100 \mathrm{~W}$. As in the previous case, the simulated width and depth of the melt pool are compared with the experimental results for different speed values. Overall, the simulation results are in acceptable agreement with the experimental results (Fig. 17). The maximum difference between the widths is equal to $15 \%$ with a scanning speed of $1 \mathrm{~m} \mathrm{~s}^{-1}$ (Fig. 17a). The comparison of the depths gives also a maximum difference of $15 \%$ when the scanning speed is equal to $0.75 \mathrm{~m} \mathrm{~s}^{-1}$ (Fig. 17b).

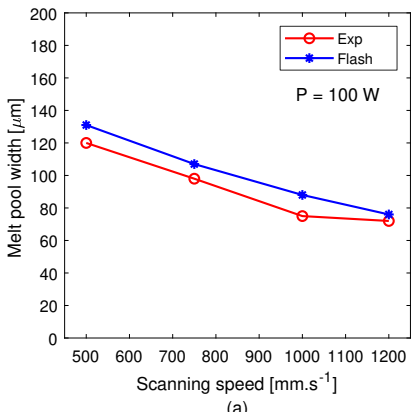

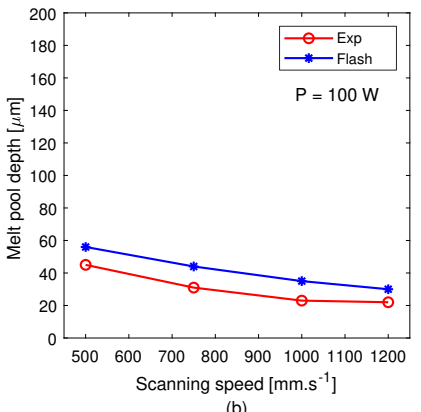

Figure 17: Flash model validation, $P=100 \mathrm{~W}$

After the model validation, it is possible to optimize the computation time and the memory size used.

\section{Optimization of the computation time}

Simulations using the Flash method require relatively small temporal and spatial discretization steps to follow the assumptions and calculation principle defined above. However, maintaining such small discretization parameters has a direct impact on the computation time and the memory size reserved for the simulation. In the example illustrated in Fig. 11, with the same simulation parameters, the computation time is $679 \mathrm{~s}$ and the memory used is equal to $271 \mathrm{MB}$.

To reduce the complexity of the problem and computation time, it is possible to reduce the size of the area of interest to its minimum. Indeed, the analysis of the simulation (Fig. 12d) shows that the major thermal change is spatially limited and close to the point of lasermaterial interaction. It is also temporally limited to moments close to the laser irradiation. For this purpose, the next section is dedicated to determine the parameters yielding the lowest computation time under the temperature simulation error constraint.

\subsection{Calculation of temporal and spatial neighbor- hoods}

The temporal neighborhood is defined as the duration of thermal effects of an elementary flash that needs to be considered in the simulation to model the global effects on the part for a given temporal $\Delta t$ and spatial discretization step $d x$. In the same way, the spatial neighborhood is defined as the maximum width of the thermal effects of an elementary flash that needs to be considered. Thus, the temporal and spatial neighborhoods are used to define, at each time step, the area where the preheating temperature should not be neglected. Thus, the threshold temperature $T_{s}$ defines the absolute error that is considered acceptable. The temporal and spatial neighborhoods can be calculated from an iso-value of the temperature field according to the following steps:

1. Simulate a scanning path segment (Fig. 18).

2. Extract the iso temperature contour line equal to the preheating temperature $T_{0}$ plus the threshold temperature $T_{s}$.

3. Calculate the spatial neighborhood that corresponds to the maximum width of the iso temperature contour line.

4. Calculate the temporal neighborhood corresponding to the length between the center of the laser spot and the center point of the first flash whose effects are mostly above the threshold temperature $T_{s}$.

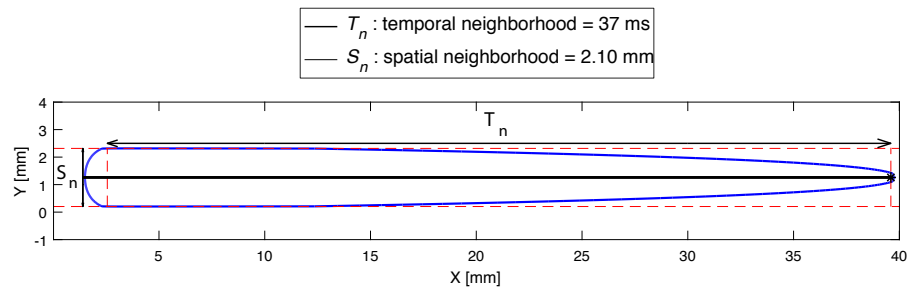

Figure 18: Calculation of temporal and spatial neighborhoods

In the example Fig. 18, the threshold temperature is set to $1 \mathrm{~K}$. The application of this method in the case of the previous example 
(Fig. 11) leads to a reduction in the amount of data to be managed during the simulation. Table 2 shows that the computation time and the memory used decrease by reducing the temporal and spatial neighborhoods. For the rest of this paper, the temporal and spatial neighborhoods are equal to $37 \mathrm{~ms}$ and $2.1 \mathrm{~mm}$, respectively, corresponding to a $1 \mathrm{~K}$ threshold.

Table 2: Variation of computation time and memory as a function of neighborhood parameters

\subsection{Parameter setting}

\subsubsection{Temporal subsampling}

To reduce the computation time further and optimize the memory reserved for simulation, it is also possible with the Flash method to calculate the thermal effects only at specific times, while maintaining a low temporal discretization. The subsampling is based on four steps:

1. Discretize the path to be simulated with a relatively small temporal step to approach the continuous behavior of the laser (Fig. 19).

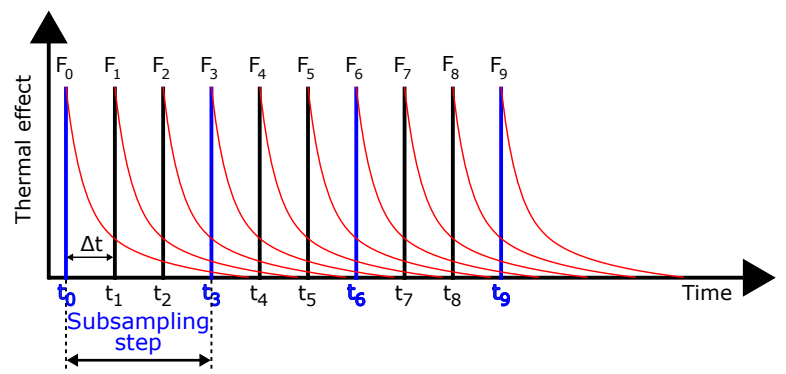

Figure 19: Diagram representing the subsampling calculation method

2. Identify the required calculation times, which are defined as a function of subsampling step $S$. This latter is a multiple of the temporal step. In the example presented in Fig. 19, it is equal to $3 \Delta t$. Thus, the calculation times are: $t_{0}, t_{3}, t_{6}, t_{9}$.

3. Identify at each calculation time the effects of flashes $F_{i}$ that have to be considered in the simulation. For example, at time $t_{6}$, the flashes that have an effect in the simulation are $F_{3}, F_{4}$, $F_{5}$, and $F_{6}$.

4. Sum the thermal effects of the considered flashes at each required calculation time.

The temporal subsampling method allows for the reduction in the computation time and memory used. It has been applied, with different subsampling step values, in the case of the simulation described in Fig. 11. Plots of corresponding computation time in
Fig. 20a and memory used in Fig. 20b highlight a high decrease from $10 \mu \mathrm{s}$ to $50 \mu \mathrm{s}$.

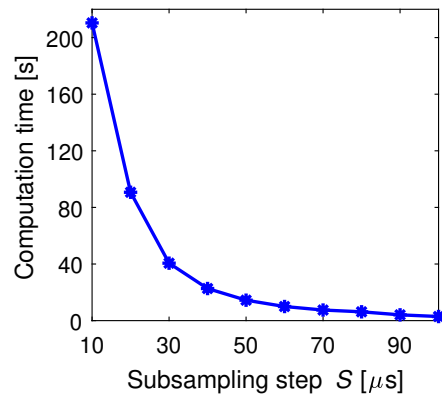

a

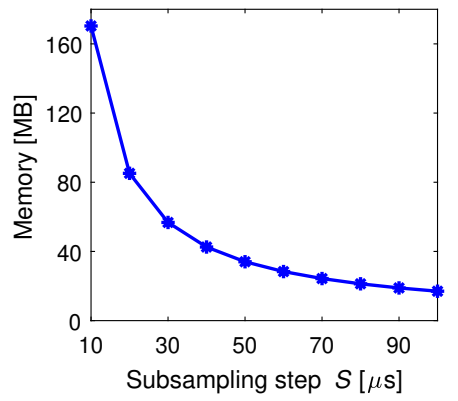

b
Figure 20: Evolution of the computation time and memory as functions of the subsampling step

\subsubsection{Setting of the discretization step}

The spatial discretization is an important parameter in thermal modeling with numerical methods. When the discretization step is small, the calculation becomes accurate and rich, especially in areas with a high variation of thermal gradients. However, simulation is more expensive in terms of computation time and memory. In the proposed method, two different types of discretization are used: the first one is used to simulate the elementary flash, while the second one concerns the discretization of the surface to be simulated. In general, the simulation is carried out with small and equal spatial steps to generate the elementary flash and the discretization of the simulated surface (Fig. 21a). This provides richer thermal mappings and more information on the temperature distribution on the surface. In addition, the points of the scanning path and the flash centers coincide with the calculation points of the surface discretization. However, this configuration increases the computation time and required memory considerably, especially in the case of large surfaces.

With the Flash method, it is possible to increase the discretization step without impacting the accuracy of results. However, the points of the scanning path may not match the points of the surface discretization. The proposed solution consists of managing two different discretizations: a finer discretization step $(10 \mu \mathrm{m})$ in the area of interest to simulate the elementary flash and a coarser discretization for the simulated surface. The discretization step $D X$ of the surface is a subsampling of the spatial step of the flash $d x$ (Fig. 21b).

Different discretization step values for the surface $D X$ are tested in the case of the previous example (Fig. 11). The spatial step $d x$ for the flash generation is kept constant $(10 \mu \mathrm{m})$. The curve presented in Fig. 22a shows the variation in computation time as a function of the discretization step of simulated surface. The increase in the discretization step size of the simulated surface reduces the computation time to about $0.9 \mathrm{~s}$ for a step equal to $200 \mu \mathrm{m}$. In addition, the increase in the discretization step reduces the memory size used (Fig. 22b). With a step size equal to $10 \mu \mathrm{m}$, the required memory 
a

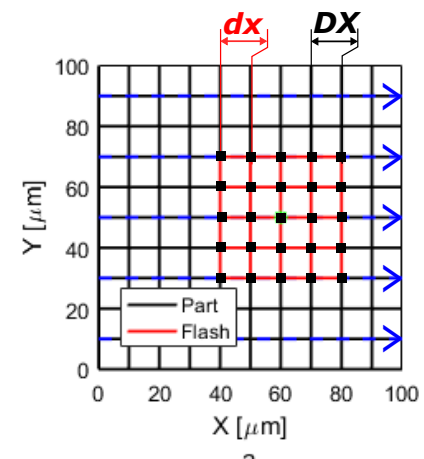

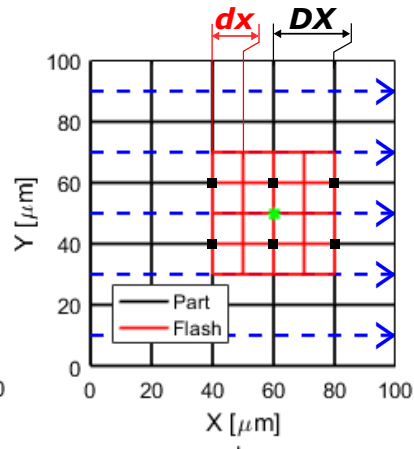

b
Figure 21: (a) Finer and (b) coarser surface discretization

size is equal to $331 \mathrm{MB}$, while this value decreases to $0.82 \mathrm{MB}$ with a discretization step equal to $200 \mu \mathrm{m}$. With this method, the number of points to qualify the thermal state of a surface is highly reduced; thus, it is possible to simulate large surfaces with reasonable computation time and required memory. To detect the main physical states that may occur during melting and identify the liquid area, it is recommended that the maximum size of a discretization step does not exceed the width of the melt pool.

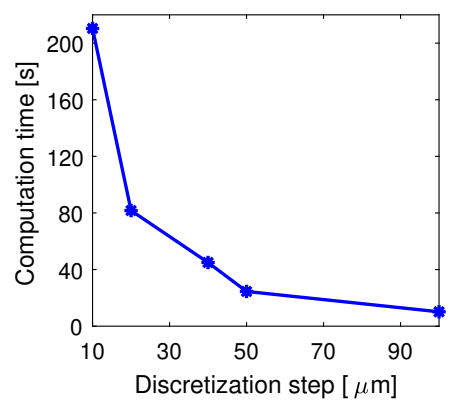

a

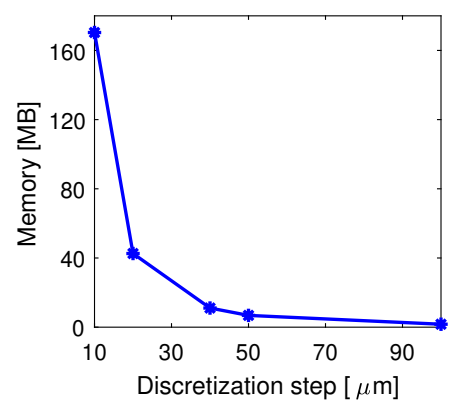

b
Figure 22: Evolution of the computation time and memory used as functions of the spatial discretization step of the simulated surface

\section{Thermal analysis of scanning paths}

\subsection{Maximum temperature}

This section studies the influence of the scanning strategies on the temperature field of a surface with a cross section of $1 \times 1 \mathrm{~mm}^{2}$. For that purpose, three scanning paths are tested: zigzag, contouring out-in scanning, and contouring in-out scanning.

To simulate the scanning paths rapidly and obtain rich and sufficient maps to evaluate the temperature distribution and thermal gradient, it is necessary to identify the optimal subsampling step and discretization step. This study has been conducted in the case of the zigzag path. Fig. 23 shows the effect of increasing the subsampling step on the maximum and minimum simulated temperature and the maximum error. The maximum error represents the maximum difference between the maximum temperature map obtained for each subsampling step compared to the one obtained by the reference step, which is equal to $10 \mu \mathrm{s}$. As expected, the analysis shows that the error increases with the subsampling step. In the same way, the lowest temperature is always close to the melting temperature.

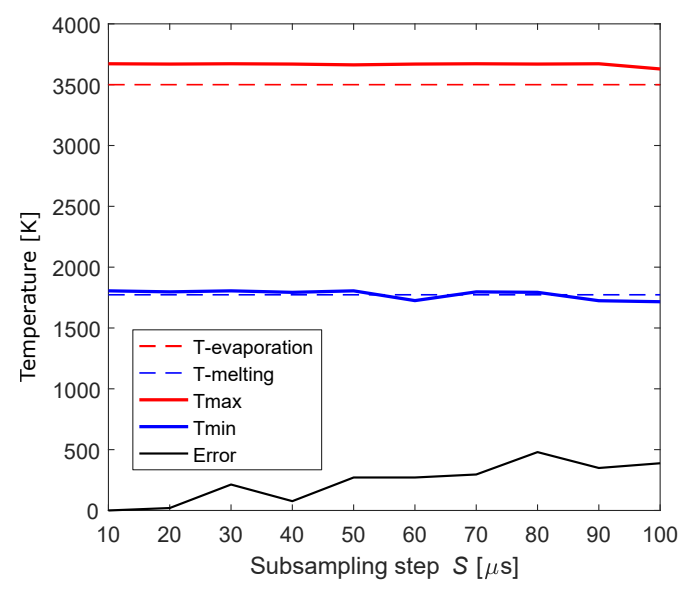

Figure 23: Evolution of the temperatures according to the subsampling step

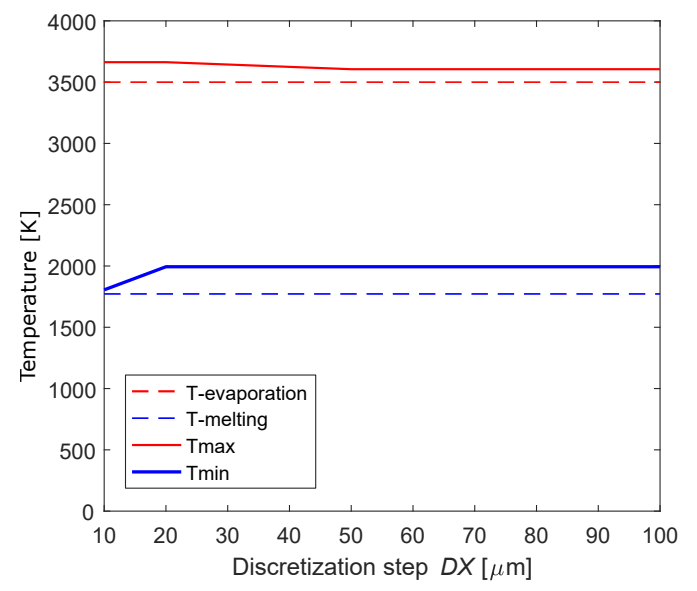

Figure 24: Evolution of the temperatures according to the spatial step

Whatever the tested subsampling step, the simulation results show the areas where the temperature is high. However, with step values greater than $50 \mu \mathrm{s}$, the temperatures are lower than the melting temperature. These unmelted points did not appear with the reference subsampling step. Therefore, the temporal resolution obtained by these values does not allow a correct thermal analysis to be performed. For this reason, the maximum value of subsampling step $S$ that can be used is equal to $50 \mu$ s.

The next phase consists of determining the discretization step. 
Table 3: Comparison between the simulation parameters for different scanning strategies

The zigzag path simulation shows that the increase in the discretization step of the simulated surface does not have a large influence on the temperature results (Fig. 24).

The temperature at all points is higher than the melting temperature. To ensure a good resolution, the maximal discretization step $D X$ is chosen to be $20 \mu \mathrm{m}$.

The three paths are simulated with the reference parameters ( $D X=10 \mu \mathrm{m}$ and $S=10 \mu \mathrm{s}$ ) and with the previously identified parameters $(D X=20 \mu \mathrm{m}$ and $S=50 \mu \mathrm{s})$. In both cases, paths are discretized with a common spatial step $d l=10 \mu \mathrm{m}$. Table 3 presents the simulation results. The developed model, with $D X=$ $20 \mu \mathrm{m}$ and $S=50 \mu \mathrm{s}$, allows the simulation of the scanning paths in a fast way and with reduced memory storage. The measurement of the maximum temperature shows that in both cases, it is possible to identify overheating areas when the vaporization temperature is reached. There is a small difference in the minimum temperature; however, the recorded values are higher than the melting temperature.

As shown in Fig. 25a and Fig. 25d, the zigzag path leads to areas of overheating at the edges. Indeed, when the laser reaches the end of a line, besides melting the local area, it constitutes an additional preheating factor for the beginning of the next line, which tends to reach a higher temperature. The measurement of the maximum temperature reached by the contouring scanning reveals the problem of overheating at the center. The temperature mapping also shows that this area is more critical when the laser scans the surface from outside to inside. (Fig. 25b and Fig. 25e). In this case, the scanning from the edge of the surface to the center isolates the heat in the inner area. This phenomenon leads to a heat accumulation in this area. In addition, there is an increase in temperature when the laser changes the scanning direction.

Overheated areas caused by some trajectories can result in the instability of the melt pool, which leads to the appearance of the

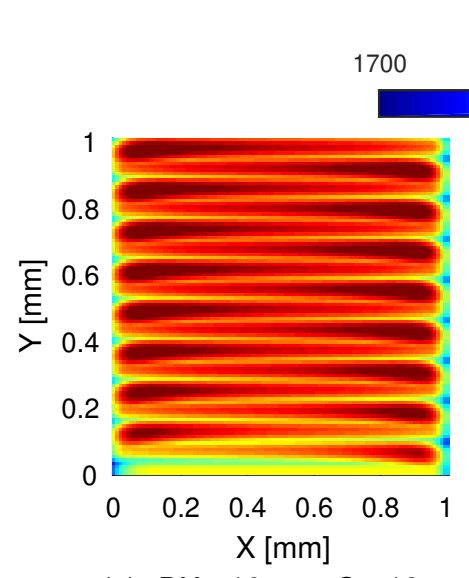

(a), $D X=10 \mu \mathrm{m}, S=10 \mu \mathrm{S}$

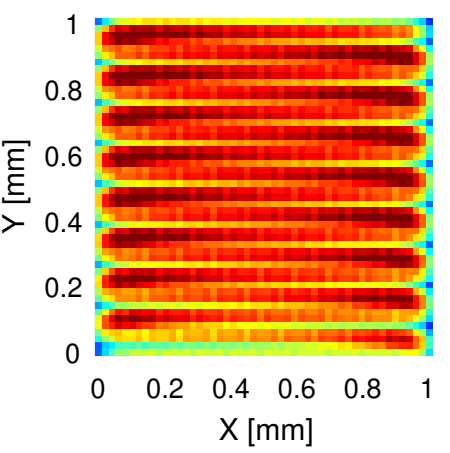

(d), $D X=20 \mu \mathrm{m}, S=50 \mu \mathrm{s}$

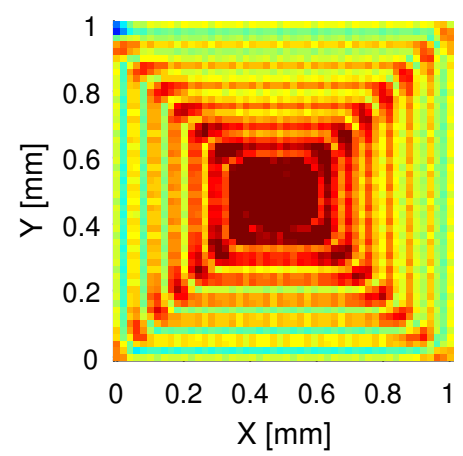

(e), $D X=20 \mu \mathrm{m}, S=50 \mu \mathrm{S}$

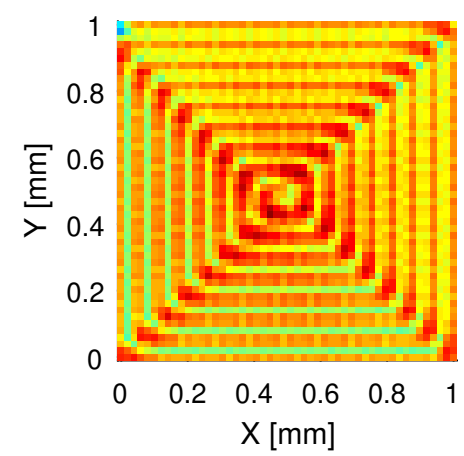

(f), $D X=20 \mu \mathrm{m}, S=50 \mu \mathrm{S}$

Figure 25: Map of maximum temperature for different scanning strategies 
Marangoni effect, and consequently, to the formation of the material bumps behind the liquid part [28]. This phenomenon can impact the melting process or even block the roller when the next layer is applied. Ultimately, the increase in temperature can lead to the vaporization of the material. The droplets escaping from the melt pool can impact the surface quality of the produced parts.

\subsection{Maximum gradient}

Spatial gradients of temperature during LPBF manufacturing cause significant plastic deformation and residual stress in the part after cooling [11]. Fig. 26 shows the maximum norm of thermal gradient $G_{\max }$ (Eq. (16)), simulated on each point of the surface over time for the three scanning paths.

$$
G_{\max }=\max _{t}(\|\vec{\nabla} T(x, y, z, t)\|)
$$

The contouring scanning presents the highest norm of the gradient (outside toward inside or inside toward outside). The laser takes a longer time to reach an already melted portion, and the temperature had enough time to decrease by conduction. Then, scanning close to an already cooled area generates an important variation in the temperature. The effect of the scanning strategy is clearly visible on the maximum norm of thermal gradient.

\section{Conclusion}

This paper presents a 3D thermal model based on an analytical method of heat diffusion. This model simulates the evolution of the temperature field and the distribution of thermal stresses during the manufacturing of a layer. Results have been validated by comparison with reference FEM software and the experimental results within a Ti6Al4V test case. The main advantage of the proposed method is the relative confidence of the results compared to the computational speed. The test campaign on the variation of temporal and spatial neighborhood and discretization parameters shows that significant improvements in computing resources are possible without significantly altering the thermal simulation results.

Using this simulation method, the thermal history of each point of the domain can be computed independently from the thermal state of the other points, and each time step is independent of the

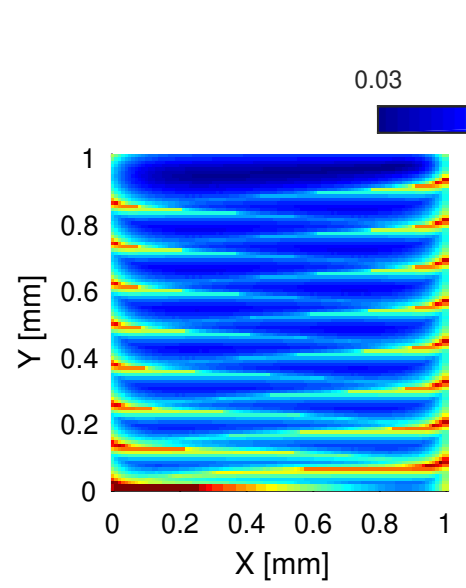

(a), $D X=10 \mu \mathrm{m}, S=10 \mu \mathrm{s}$

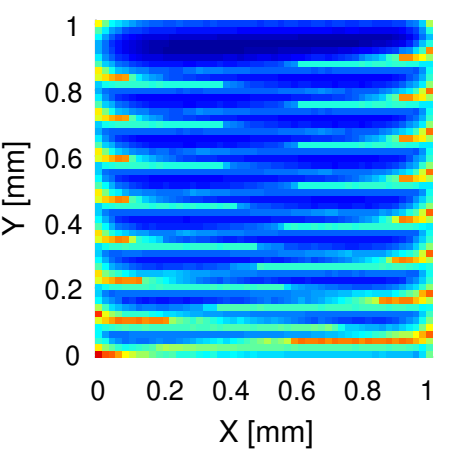

(d), $D X=20 \mu \mathrm{m}, S=50 \mu \mathrm{s}$

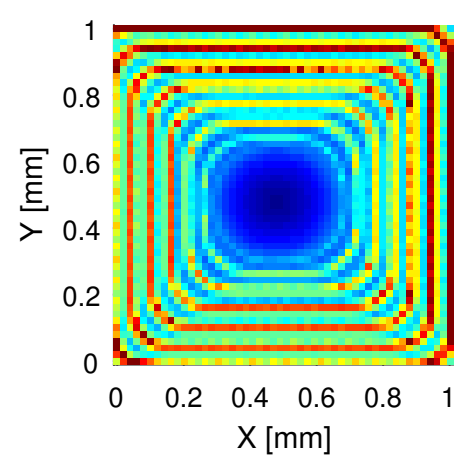

(e), $D X=20 \mu \mathrm{m}, S=50 \mu \mathrm{s}$

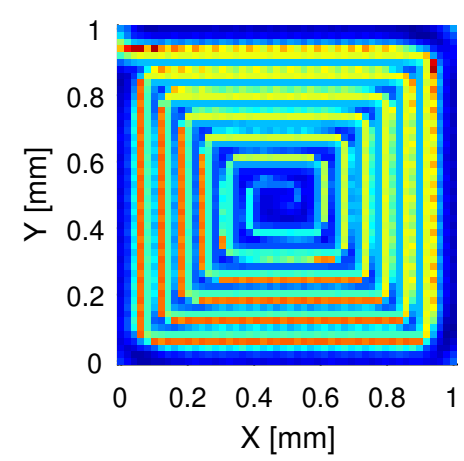

(f), $D X=20 \mu \mathrm{m}, S=50 \mu \mathrm{s}$

Figure 26: Map of maximum thermal gradient for different scanning strategies 
other step. This allows for parallelization of the simulation and thus further improvement of the computing speed. In future work, our intention is to exploit mathematical techniques such as convolution operations using GPU to further reduce computation time and memory occupancy and thus be able to simulate larger parts.

\section{Acknowledgments}

This work is supported by the SOFIA project and funded by Bpifrance.

\section{References}

[1] Kempen, K., Thijs, L., Vrancken, B., Buls, S., Humbeeck, J. V. and Kruth, J. P. (2013), "Producing crack-free, high density M2 HSS parts by Selective Laser Melting: Pre-heating the baseplate", 24th International SFF Symposium - An Additive Manufacturing Conference, SFF 2013, pp. 131-139.

[2] Jhabvala, J., Boillat, E., Antignac, T. and Glardon, R. (2010), "On the effect of scanning strategies in the selective laser melting process", Virtual and Physical Prototyping, Vol. 5 No. 2, pp. 99-109, doi:99109.10.1080/17452751003688368.

[3] Buchbinder, D., Meiners, W., Pirch, N. and Wissenbach, K. (2014), "Investigation on reducing distortion by preheating during manufacture of aluminium components using selective laser melting", Journal of Laser Applications, Vol. 26 No. 1, doi:10.2351/1.4828755.

[4] Beal, V. E., Erasenthiran, P., Hopkinson, N., Dickens, P. and Ahrens, C. H. (2008), "Scanning strategies and spacing effect on laser fusion of $\mathrm{H} 13$ tool steel powder using high power Nd:YAG pulsed laser", International Journal of Production Research, Vol. 46 No. 1, pp. 217-232, doi:10.1080/00207540500168279.

[5] Yang, Y., Loh, H. T., Fuh, J. Y. H. and Wang, Y. (2002), "Equidistant path generation for improving scanning efficiency in layered manufacturing", Rapid Prototyping Journal, Vol. 8 No. 1, pp. 30-37, doi:10.1108/13552540210413284.

[6] Chantarapanich, N., Laohaprapanon, A., Wisutmethangoon, S., Jiamwatthanachai, P., Chalermkarnnon, P., Sucharitpwatskul, S., Puttawibul, P. and Sitthiseripratip, K. (2014), "Fabrication of three-dimensional honeycomb structure for aeronautical applications using selective laser melting: a preliminary investigation", Rapid Prototyping Journal, Vol. 20 No. 6, pp. 551-558, doi:10.1108/RPJ-08-2011-0086.

[7] Mercelis, P. and Kruth, J. P. (2006), "Residual stresses in selective laser sintering and selective laser melting", Rapid Prototyping Journal, Vol. 12 No. 5, pp. 254-265, doi:10.1108/13552540610707013.

[8] Ma, L. and Bin, H. (2006), "Temperature and stress analysis and simulation in fractal scanning-based laser sintering", The International Journal of Advanced Manufacturing Tech- nology, Vol. 34 No. 9, pp. 898-903, doi:10.1007/s00170-0060665-5.

[9] Luo, Z. and Zhao, Y. (2018), "A survey of finite element analysis of temperature and thermal stress fields in powder bed fusion Additive Manufacturing", Additive Manufacturing, Vol. 21, pp. 318-331, doi:10.1016/j.addma.2018.03.022.

[10] Criales, L. E., Arisoy, Y. M. and Ozel, T. (2016), "Sensitivity analysis of material and process parameters in finite element modeling of selective laser melting of Inconel 625", The International Journal of Advanced Manufacturing Technology, Vol. 86 No. 9, pp. 2653-2666, doi:10.1007/s00170015-8329-y.

[11] Hussein, A., Hao, L., Yan, C. and Everson, R. (2013), "Finite element simulation of the temperature and stress fields in single layers built without support in selective laser melting", Materials \& Design, Vol. 52, pp. 638-647, doi:10.1016/j.matdes.2013.05.070.

[12] Li, C., Fu, C., Guo, Y. B. and Fang, F. (2015), "Fast Prediction and Validation of Part Distortion in Selective Laser Melting", Procedia Manufacturing, Vol. 43, pp. 8-12, doi:10.1016/j.promfg.2015.09.042.

[13] Nickel, A. H., Barnett, D. M. and Prinz, F. B. (2001), 'Thermal stresses and deposition patterns in layered manufacturing", Materials Science and Engineering, Vol. 317 No. 1, pp. 59-64, doi:10.1016/S0921-5093(01)01179-0.

[14] Foroozmehr, E. and Kovacevic, R. (2010), 'Effect of path planning on the laser powder deposition process: thermal and structural evaluation", The International Journal of Advanced Manufacturing Technology, Vol. 51 No. 5, pp. 659669, doi:10.1007/s00170-010-2659-6.

[15] Rosenthal, D. (1941), "Mathematical Theory of Heat Distribution during Welding and Cutting", Welding Journal, Vol. 20 No. 5, pp. 220-234.

[16] Promoppatum, P., Yao, S. C., Pistorius, S. C. and Rollett, A. D. (2017), "A comprehensive comparison of the analytical and numerical prediction of the thermal history and solidification microstructure of Inconel 718 products made by laser powder-bed fusion", Engineering, Vol. 3 No. 5, pp. 685-694.

[17] Plotkowski, A., Kirka, M. M. and Babu, S. S. (2017), "Verification and validation of a rapid heat transfer calculation methodology for transient melt pool solidification conditions in powder bed metal additive manufacturing", Additive Manufacturing, Vol. 18, pp. 256-268.

[18] Forslund, R., Snis, A. and Larsson, S. (2018), "Analytical solution for heat conduction due to a moving Gaussian heat flux with piecewise constant parameters", Materials (Basel), Vol. 12, pp. 227-240, doi:10.1016/j.apm.2018.09.018.

[19] Yang, Y., Knol, M. F., Keulen, F. V. and Ayas, C. (2018), "A semi-analytical thermal modelling approach for selective laser melting", Additive Manufacturing, Vol. 21, pp. 284297.

[20] ESI, "Engineering Systems International, Metallic Additive Manufacturing Process Simulations", https://www.esi-group.com/software-solutions/virtual- 
manufacturing/additive-manufacturing.

[21] Vastola, G., Pei, Q. X. and Zhang, Y. W. (2018). "Predictive model for porosity in powder-bed fusion additive manufacturing at high beam energy regime", Additive Manufacturing, Vol. 22, pp. 817-822, doi:10.1016/j.addma.2018.05.042.

[22] Mukherjee, T. and DebRoy, T. (2018). "Mitigation of lack of fusion defects in powder bed fusion additive manufacturing", Journal of Manufacturing Processes, Vol. 36, pp. 442-449, doi: 10.1016/j.jmapro.2018.10.028.

[23] Cernuschi, F., Russ, A. and Lorenzoni, L. (2001), "In-plane thermal diffusivity evaluation by infrared thermography", Journal of Applied Physics, Vol. 72 No. 10, pp. 3988-3995, doi:10.1063/1.1400151.

[24] Dai, D. and Gu, D. (2014), "Thermal behavior and densification mechanism during selective laser melting of copper matrix composites: Simulation and experiments", Materials \& Design, Vol. 55, pp. 482-491, doi:doi.org/10.1016/j.matdes.2013.10.006.

[25] Dilip, J. J. S., Zhang, S., Teng, C., Zeng, K., Robinson, C., Pal, D. and Stucker, B. (2017), 'Influence of processing parameters on the evolution of melt pool, porosity, and microstructures in Ti6Al4V alloy parts fabricated by selective laser melting", Progress in Additive Manufacturing, Vol. 2 No. 3, pp. 157-167, doi: 10.1007/s40964-017-0030-2.

[26] Olleak, A. A. end Xi, Z. (2018), "Finite element modeling of the selective laser melting process for Ti6Al4V", Proceedings of the 29th Annual International Solid Freeform Fabrication Symposium - An Additive Manufacturing Conference, At Austin, TX, pp. 1710-1720.

[27] Gharbi, M., Peyre, P., Gorny, C., Carin, M., Morville, S., Masson, P. L., Carron, D. and Fabbro, R. (2013), "Influence of various process conditions on surface finishes induced by the direct metal deposition laser technique on a Ti6A14V alloy", Journal of Materials Processing Technology, Vol. 213 No. 5, pp. 791-800, doi: doi.org/10.1016/j.jmatprotec.2012.11.015.

[28] Dai, D. and Gu, D. (2015), "Tailoring surface quality through mass and momentum transfer modelling using a volume of fluid method in selective laser melting of TiC/AlSi10Mg powder", International Journal of Machine Tools and Manufacture, Vol. 88, pp. 95-107, doi:10.1016/j.ijmachtools.2014.09.010.

[29] Parker, W. J., Jenkins, R. J., Butler, C. P. and Abbott, G. L. (1961), "Flash Method of Determining Thermal Diffusivity, Heat Capacity, and Thermal Conductivity", Journal of Applied Physics, Vol. 32 No. 9, pp. 1679-1684, doi:10.1063/1.1728417. 